\title{
An experimental approach to analyze aerosol and splatter formation in a dental practice
}

\author{
P. Mirbod ${ }^{1 *}$, E. A. Haffner ${ }^{1}$, M. Bagheri ${ }^{1}$, J. E. Higham ${ }^{2}$ \\ ${ }^{1}$ University of Illinois at Chicago, Department of Mechanical and Industrial Engineering, 842 W. Taylor \\ Street, Chicago, IL, 60607, USA \\ ${ }^{2}$ University of Liverpool, School of Environmental Sciences, Liverpool, UK \\ *pmirbod@uic.edu
}

\begin{abstract}
The flow velocity, trajectories, and size distribution of droplets produced during a dental scaling procedure using a Cavitron ultrasonic scalar (CUS) has been investigated by optical flow tracking velocimetry and shadowgraphy measurements. The droplet sizes are found to vary from $5-500 \mu \mathrm{m}$; these correspond to droplet nuclei that could carry viruses. The droplet velocities also vary between $0.7 \mathrm{~m} / \mathrm{s}$ and $1.3 \mathrm{~m} / \mathrm{s}$. These observations confirm the critical role of aerosols in the transmission of disease during dental procedures, providing invaluable knowledge for developing protocols and procedures to ensure the safety of both dentists and patients especially during COVID-19 pandemic.
\end{abstract}

\section{Introduction}

Global emergence of novel COVID-19 virus has required health care professionals to implement prescriptive adjustments. One of the high-risk areas of infection is dental offices since many of dental procedures produce aerosols because of high-speed dental instruments Harrel and Molinari (2004). This lead the researchers to design , e.g., Aero-Shields, barriers and high-powered suction equipment, to reduce the level of aerosol particle concentration and protect clinicians Liu et al. (2019), and Majidi and Club (2020). To design the appropriate safety tools, it is crucial to understand how this virus, along with others, propagate throughout the atmosphere of the exam room. This plan is to perform experiments on the measurement of droplet sizes in the splatter created from a patient's mouth by a Cavitron Ultrasonic Scaler (CUS) using optical flow tracking velocimetry (OFTV) and Shadowgraphy techniques. This study is the first of its kind to investigate droplet nuclei size and velocity distribution in detail using quantitative fluid mechanics methods with the implementation of dental instruments. The obtained data in this work can be employed to quantify the amount of virus transmitted to a poorly ventilated space in a dental clinic. In addition, they can be used to further model how airborne particles are transported into the human respiratory system.

\section{Materials and Methods}

OFTV (optical flow tracking velocimetry) is a commonly used method in fluid mechanics Fullmer et al. (2020) that we used it to determine the velocity fields and Lagrangian paths of droplets created by the Cavitron Select SPS Ultrasonic Scaler (CUS); Using a $1 \mathrm{~mm}$ laser sheet created by a $527 \mathrm{~nm}$ Nd-YLF (Photonics Industries, DM20-527) laser. We were able to illuminate a single plane inside the aerosol, providing us with a two-dimensional (2D) slice for analysis. Using a high-speed camera (Phantom) equipped with a Nikon lens with a focal length of $60 \mathrm{~mm}$, we were further captured the reflections from the water droplets. We considered two different planes, (P1 parallel, and P2 perpendicular to the tip of the CUS), as shown in Fig. $1(b, c)$. In each case, to ensure that the mean velocities were fully resolved, 3000 images were collected resolving more than 100 integral time scales. As in a dental practice, the tip of the Slimline placed perpendicular to the front lower teeth pointing towards the gum line (Fig. 1a) for all of the experiments where the CUS connected to a standard water tap (i.e., 20 psi to 40 psi pressure). The measured flow rate defined as $29.5 \mathrm{ml} / \mathrm{min}$. 

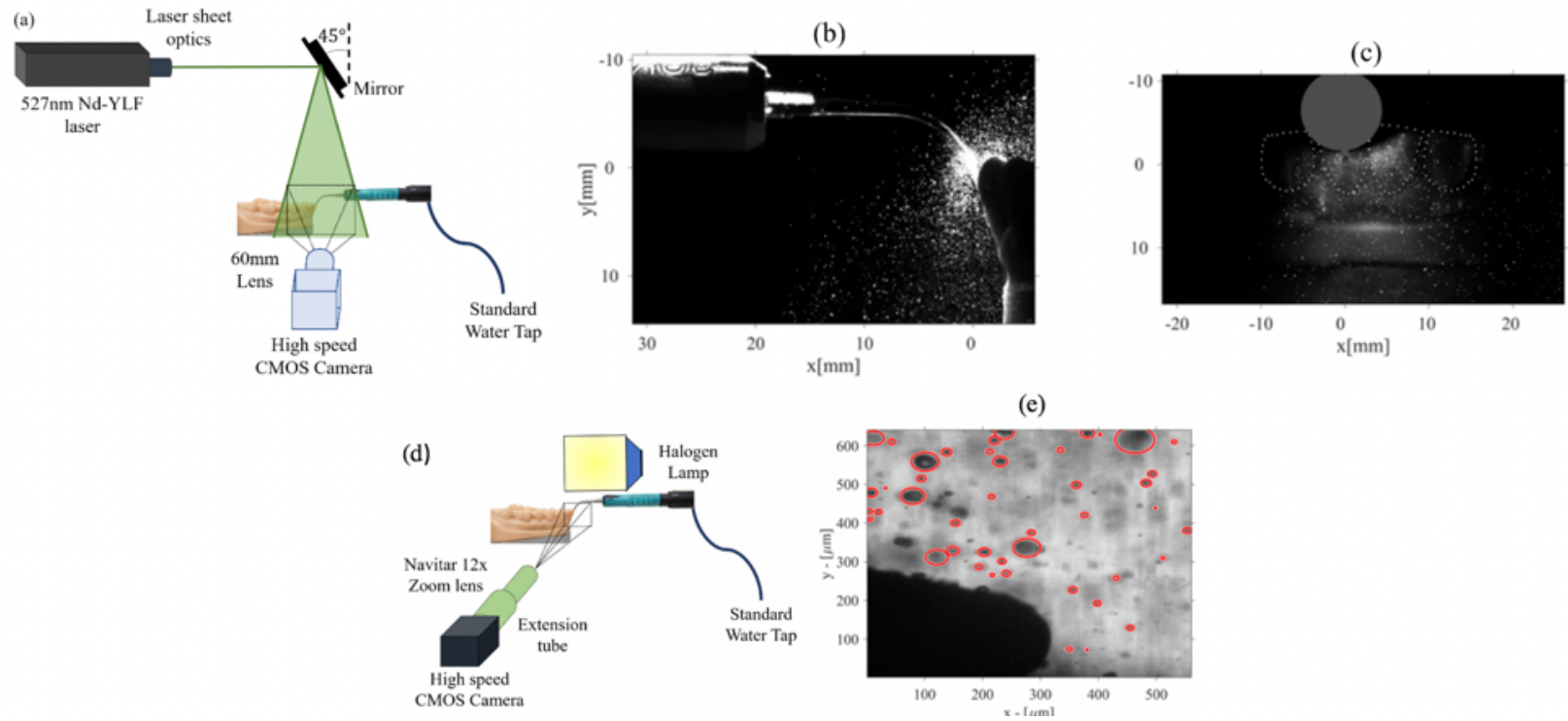

Figure 1: (a) Setup schematic for the optical flow tracking velocimetry (OFTV) technique to detect the droplet velocity and lagrangian path. Examples of the raw OFTV images in the (b) P1 plane and (c) P2 plane recorded with a high-speed camera at $7.6 \mathrm{kHz}$. (d) Schematic of the experimental setup of backlight illumination for the shadowgraphy technique. (e) An example of backlight illumination at $0.08 \mathrm{~s}$ recorded using a high-speed camera at $7.6 \mathrm{kHz}$ and two halogen backlights.

We also used shadowgraphy method which is a well-used technique in fluid mechanics to quantitatively visualize small droplets using simple optics Settles (2001). To create these visualizations, we used a high magnification and back-light illumination (Fig. 1d). The measurement plane is defined by the camera depth of focus in the focal plane. A Navitar zoom lens (Thorlabs, Inc.) used attached to the high-speed Phantom camera set to an exposure rate of $20 \mu \mathrm{s}$. This allowed us to zoom into a small region ( $100 \mu \mathrm{m}$ thick) to accurately measure the size of the droplets on the order of $5 \mu \mathrm{m}$ (see Fig. 1e). From the raw images, we then used an in-house code to detect the particles and determine the size and location of each droplet. The code works by first binarizing the raw image based on an adaptive threshold, and an adaptive Hough transform Illingworth and Kittler (1987), we then determined the circular regions, i.e., droplets, and defined the velocity of the droplets using OFTV method. However, instead of using the eigenfeatures for droplet detection, we employed the centroids determined by the Hough transform Mirbod et al. (2021).

To generalize the problem and to characterize the breakup of the fluid in more detail, we also examined some dimensionless parameters, for which we measured the characteristic length to be the CUS tip diameter, $\mathrm{d}=635 \mu \mathrm{m}$. Comparing these dimensionless values with those produced by coughing and sneezing Bourouiba et al. (2014) reveals that some of the values reported in this study (e.g., Reynolds number) are still lower compared to the turbulent flow produced by coughing/sneezing, while the Stokes number due to the droplet formed by CUS is much higher compared to coughing/sneezing cases meaning that most of the droplets settle down. However, our data shows that droplet size in the dental procedure are much smaller in size where precautions must be considered for the safety of those who are exposed to these droplets. More details can be found in Mirbod et al. (2021) with the typographical errors in the table that are fixed herein.

\begin{tabular}{cccccccc}
\hline & $\begin{array}{c}\text { Flow rate } \\
(\mathrm{ml} / \mathrm{min})\end{array}$ & $\begin{array}{c}\boldsymbol{u}_{\boldsymbol{o}} \\
(\mathrm{cm} / \mathbf{s})\end{array}$ & $\boldsymbol{R e}$ & We & Oh & $\overline{\boldsymbol{\tau}}_{\boldsymbol{o}}$ & St \\
\hline $\begin{array}{c}\text { Aerosol } \\
\text { formation by } \\
\text { the CUS }\end{array}$ & 29.5 & 155.25 & 982.50 & 20.97 & $4.7 \times 10^{-3}$ & $7.55 \times 10^{-3}$ & 18.46 \\
\hline
\end{tabular}

Table 1: The experimental parameters for the different tests conducted at $20^{\circ} \mathrm{C}$. The dimensionless parameters remained constant throughout all experiments 


\section{RESULTS}

Fig. 2 shows the global velocity components (U, V, and magnitude) for the planes both parallel and perpendicular to the teeth, i.e., planes P1 and P2, respectively, obtained from OFTV. Figs. 2(a, d) demonstrate the maximum $U$ velocity of $1.5 \mathrm{~m} / \mathrm{s}$ that occurs near the tip of the CUS close to the front teeth, while it is reduced far from the tip. The mean $\mathrm{V}$ velocity is the lowest at $0.2 \mathrm{~m} / \mathrm{s}$ compared to the mean maximum velocity $(2.5 \mathrm{~m} / \mathrm{s})$ of the magnitude velocity profile as can be seen in Fig. 2(b,c,e,f).
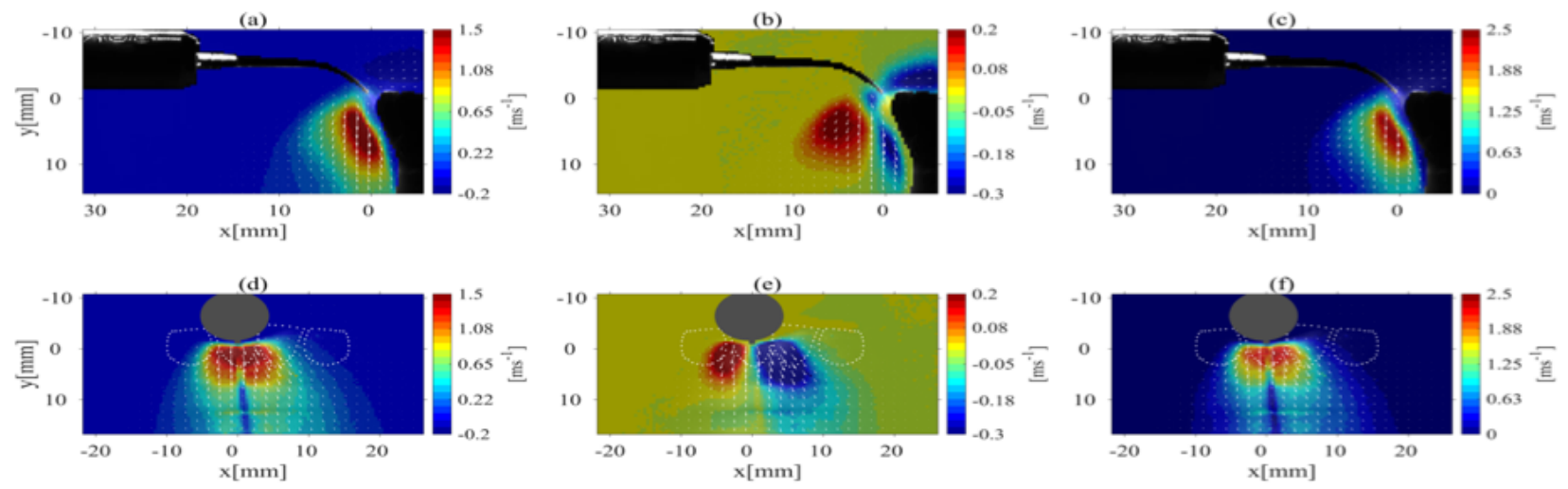

Figure 2: The mean field of the (a) V (y-axis) velocity component, (b) $\mathrm{U}$ (x-axis) velocity component, and (c) velocity magnitude for the plane parallel to the CUS tip, P1, and the (d) V (y-axis) velocity component, (e) $\mathrm{U}$ (x-axis) velocity component, and (f) velocity magnitude (y-axis) for the plane perpendicular to the CUS tip, P2. The white arrows in the figures specify the velocity vectors.

Fig. 3(a,b), shows measured droplet sizes ranges from $5 \mu \mathrm{m}$ to $500 \mu \mathrm{m}$ with a maximum observed speed of $1.4 \mathrm{~m} / \mathrm{s}$. The experimentally obtained data are in agreement with the Rosin-Rammler equation Rosin (1933) which is a well-known distribution function and a convenient representation of the droplet size distribution for liquid sprays. In particular, this fitting can be used as an initial condition in numerical simulations to further model how droplets are transmitted to the environment, including dental clinics/offices.
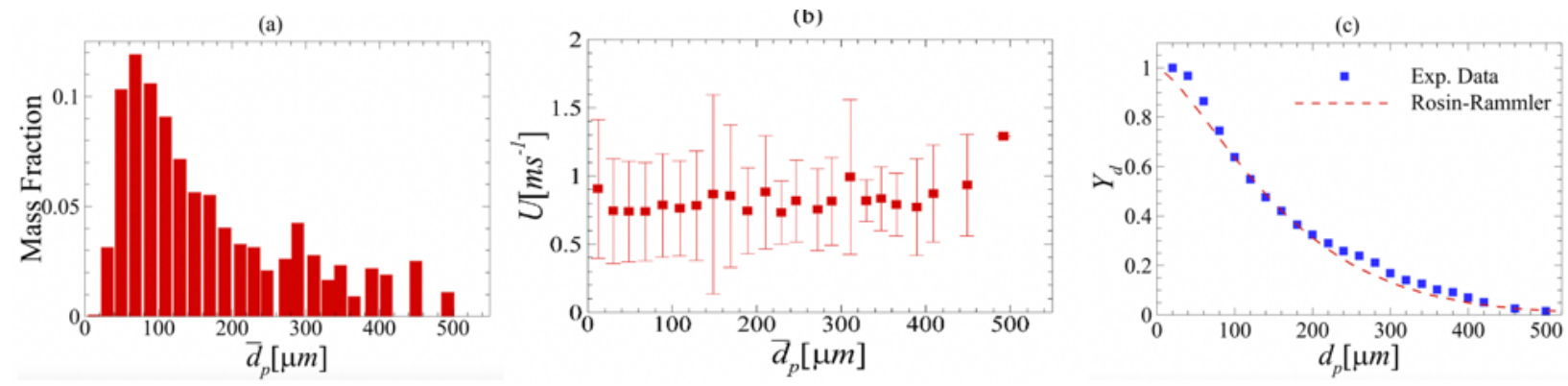

Figure 3: (a) Histogram of the droplet size distribution, (b) the velocity distribution of the droplets, and (c) the Rosin-Rammler curve fitted for our obtained experimental droplet size data with a $29.5 \mathrm{ml} / \mathrm{min}$ flow rate.

\section{Acknowledgements}

This work was funded by the University of Illinois at Chicago (UIC) - College of Dentistry (grant no. 200258-323152). 


\section{References}

Bourouiba L, Dehandschoewercker E, and Bush JW (2014) Violent expiratory events: on coughing and sneezing. Journal of Fluid Mechanics 745:537-563

Fullmer WD, Higham JE, LaMarche CQ, Issangya A, Cocco R, and Hrenya CM (2020) Comparison of velocimetry methods for horizontal air jets in a semicircular fluidized bed of geldart group d particles. Powder Technology 359:323-330

Harrel SK and Molinari J (2004) Aerosols and splatter in dentistry: a brief review of the literature and infection control implications. The Journal of the American Dental Association 135:429-437

Illingworth J and Kittler J (1987) The adaptive hough transform. IEEE Transactions on Pattern Analysis and Machine Intelligence pages 690-698

Liu MH, Chen CT, Chuang LC, Lin WM, and Wan GH (2019) Removal efficiency of central vacuum system and protective masks to suspended particles from dental treatment. PLoS One 14:e0225644

Majidi K and Club HD (2020) Dental clinic aerosol management with aero-shield

Mirbod P, Haffner EA, Bagheri M, and Higham JE (2021) Aerosol formation due to a dental procedure: insights leading to the transmission of diseases to the environment. Journal of the Royal Society Interface 18:20200967

Rosin P (1933) Laws governing the fineness of powdered coal. Journal of Institute of Fuel 7:29-36

Settles GS (2001) Schlieren and shadowgraph techniques: visualizing phenomena in transparent media. Springer Science \& Business Media 